\title{
EMMI Rapid Reaction Task Force Meeting on "Quark Matter in Compact Star"
}

\author{
Michael Buballa, ${ }^{1}$ Veronica Dexheimer, ${ }^{2}$ Alessandro Drago, ${ }^{3}$ Eduardo \\ Fraga, ${ }^{4,5,6}$ Pawel Haensel, ${ }^{7}$ Igor Mishustin, ${ }^{5}$ Giuseppe Pagliara, ${ }^{3}$ Jürgen \\ Schaffner-Bielich, ${ }^{4}$ Stefan Schramm, ${ }^{5}$ Armen Sedrakian, ${ }^{4}$ and Fridolin Weber ${ }^{8,9}$ \\ ${ }^{1}$ Institut für Kernphysik (Theoriezentrum), \\ Technische Universität Darmstadt, D-64289 Darmstadt, Germany \\ ${ }^{2}$ Department of Physics, Kent State University, Kent, OH 44242, USA \\ ${ }^{3}$ Dip. di Fisica e Scienze della Terra dell'Università di \\ Ferrara and INFN Sez. di Ferrara, I-44100 Ferrara, Italy \\ ${ }^{4}$ Institut für Theoretische Physik, Goethe Universität, \\ D-60438 Frankfurt am Main, Germany \\ ${ }^{5}$ Frankfurt Institute for Advanced Studies, \\ Goethe University, D-60438 Frankfurt am Main, Germany \\ ${ }^{6}$ Instituto de Fisica, Universidade Federal do Rio de Janeiro, Rio de Janeiro, RJ, Brazil \\ ${ }^{7}$ N. Copernicus Astronomical Center, Polish Academy of Sciences, \\ Bartycka 18, PL-00-716 Warszawa, Poland \\ ${ }^{8}$ Department of Physics, San Diego State University, San Diego, CA 92182-1233, USA \\ ${ }^{9}$ Center for Astrophysics and Space Sciences, \\ University of California, San Diego, La Jolla, CA 92093, USA
}

\begin{abstract}
The recent measurement of two solar mass pulsars has initiated an intense discussion on its impact on our understanding of the high-density matter in the cores of neutron stars. A task force meeting was held from October 7-10, 2013 at the Frankfurt Institute for Advanced Studies to address the presence of quark matter in these massive stars. During this meeting, the recent observational astrophysical data and heavy-ion data was reviewed. The possibility of pure quark stars, hybrid stars and the nature of the QCD phase transition were discussed and their observational signals delineated.
\end{abstract}




\section{MOTIVATION}

The recent observations of the pulsars PSR J1614-2230 and PSR J0348+0432 proofs beyond doubt the existence of neutron stars with masses close to two solar masses [1, 2]. Moreover, there are hints that there might exist even more massive neutron stars, e.g. from a recent analysis of the black-widow pulsar PSR J1311-3430 [3] and pulsar PSR J1816+4510 [4].

In addition, future astrophysical instruments will set much more refined constraints on the properties of neutron stars. The future Five hundred meter Aperture Spherical Telescope FAST and the Square Kilometre Array SKA will be sensitive enough to find an order of magnitude more millisecond pulsars and pulsars in binary systems than those found up to now, increasing the number of pulsar mass measurements accordingly [5, 6]. The gravitational wave detectors advanced LIGO and advanced VIRGO, both starting operation in 2014, will have an order of magnitude increase in their sensitivity to find gravitational waves from compact binary mergers with a projected rate of measuring about several events per

year [7]. Such increased sensitivity will allow scientists to determine the basic properties of binary neutron star systems to within a few percent [8]. Finally, future X-ray satellite missions such as NICER [9] and LOFT [10 12] plan to measure the radius of compact stars to unprecedented precision.

In reaction to these astrophysical observations and future prospects, it seems timely to discuss the role of quark matter in the cores of neutron stars. We do that by investigating quark matter properties that are relevant for compact stars and the possible impact of quark matter on astrophysical observations. A rapid reaction task force meeting of the ExtreMe Matter Institute (EMMI) on "Quark Matter in Compact Stars" was held from October 7-10, 2013 at the Frankfurt Institute for Advanced Studies (FIAS) in Frankfurt/Main, Germany. During the task force meeting, the properties of dense quark matter relevant for compact star physics were discussed in intense closed working sessions. A previous meeting of this kind focussing on the role of hyperons in neutron stars and the problem of low maximum masses of compact stars in microscopic models with hyperons, Hyperons2012, was held in Warsaw in November of 2012.

Some of the key questions addressed in the task force meeting on quark matter in compact stars were 
- what are the properties of dense matter in the core of massive pulsars with two solar masses?

- can one constrain effective models of dense QCD matter?

- is there still room for quark matter to be present in neutron stars?

- would the observation of a say 2.4 solar mass neutron star exclude exotic matter in its core?

- is there a smoking gun for the presence of quark matter in compact stars?

- are quark stars still a viable possibility? Can quark stars and hadronic stars coexist?

- what can we learn from radius measurements about the properties of QCD matter at extreme densities?

The theoretical investigation of these questions is related to two main research themes: the structure and dynamics of neutron star matter, and the properties of the quark-gluon plasma and the phase structure of strongly interacting matter. The properties of dense matter in the core of a neutron star determine the global properties of compact stars, i.e. the mass-radius relation, which was a key issue of the task force meeting. This is also relevant for the investigation of the QCD phase diagram and its possible phase transitions at high densities and low

temperatures. Possible cross relations of these two research areas were unavoidably part of the scientific discussion of the task force meeting. The presentations are available at the task force website at https://indico.gsi.de/conferenceTimeTable.py?confId=2420\#all.detailed.

\section{QUARK MATTER IN COMPACT STARS}

\section{A. Observational Astrophysical Data}

Measurements of pulsar masses in binaries provide most valuable information on the underlying equation of state (EoS) of star matter because they, being deduced from binary system parameters, are model independent within a given theory of gravity. An example of this is the recent discovery of a compact star with a mass of $1.97 M_{\odot}$, which sets an observationally "clean" lower bound on the maximum mass of a compact star via the measured 
Shapiro delay [1], spurred an intensive discussion of the phase structure of dense matter in compact stars consistent with this limit. The Shapiro delay was detected in the data of Ref. [1] with extremely high significance. In this work, robust error estimates were obtained using a Markov chain Monte Carlo (MCMC) approach to explore effectively all fit dimensions simultaneously. From the Shapiro delay, the companion white dwarf mass was deduced to be $0.500 \pm 0.006 M_{\odot}$, which suggests that the companion is a helium-carbon-oxygen white dwarf. The fits to the data also suggest that the binary system is highly edge-on with respect to the observer with an inclination of $89.17^{\circ} \pm 0.02^{\circ}$. The amplitude of the Shapiro delay increases strongly with increasing binary inclination. The fortunate combination of the high orbital inclination and massive white dwarf companion result in a Shapiro delay amplitude which is orders of magnitude larger than for most other millisecond pulsars with smaller mass companions (typically $0.1-0.2 M_{\odot}$ ) and smaller inclinations angles. The knowledge of the companion mass combined with the standard Keplerian orbital parameters and the assumption that the binary is correctly described by general relativity determine the pulsars mass to be $1.97 \pm 0.04 M_{\odot}$.

Unfortunately, the Shapiro delay mechanism does not provide information on the radius of the pulsar. Pulsar radii have been extracted, e.g., from modeling the X-ray binaries under certain reasonable model assumptions, but the uncertainties are large. A recent example is the pulse phase-resolved X-ray spectroscopy of PSR J0437-4715, the nearest known millisecond pulsar [13]. The continuum emission has at least three thermal components; the highest-temperature radiation is naturally originating from the hot magnetic polar caps of the neutron star, whereas the low-temperature emission components originates from the bulk of the surface. The pulse phase-resolved X-ray spectroscopy of PSR J0437-4715 sets a lower limit on the radius of a $1.76 M_{\odot}$ solar mass compact star of $R>11 \mathrm{~km}$ within a $3 \sigma$ error.

A second measurement of a massive compact star was reported in April 2013 by Antoniadis et al. [2]. The measurement method differs from the Shapiro delay mechanism and thus provides a model-independent confirmation of the existence of massive compact stars. To measure the mass of the pulsar, researchers studied in detail the spectrum of the companion white dwarf using observational data from the VLT. The measured radial velocity of the companion combined with the orbital fit, provided the ratio of the masses of the pulsar and the white dwarf to be $11.70 \pm 0.13$. Further, the mass of the white dwarf was deduced by 
modeling the cooling evolution, which yields for the mass of the white dwarf $0.165-0.185 M_{\odot}$ at $99.73 \%$ confidence. From the obtained white dwarf mass and the observed mass ratio $q$, one then finds that the neutron star mass is in the range of $1.97-2.05 M_{\odot}$ at $68.27 \%$, or $1.90-2.18 M_{\odot}$ at $99.73 \%$ confidence. The authors also measured the orbital decay $\dot{P}_{b}$. Given this quantity, the value of $q$, and assuming that General Relativity (GR) is the correct theory of gravity, they obtained for the pulsar mass $2.07 \pm 0.20 M_{\odot}$ at $68.27 \%$ confidence. Furthermore, the error in this measurement could be narrowed down substantially within the next couple of years using improved measurements of $\dot{P}_{b}$.

Larger than two solar mass pulsars were claimed to be observed with lower confidence in black-widow systems [3, 14]. Van Kerkwijk et al. claim that, most likely, PSR B1957+20 is a massive neutron star, with a mass of $2.40 M_{\odot}$. Their results depend among other things on the lightcurve modelling of the companion, which is based on a number of conservative assumptions, as for example, no heat transport over the surface of the star. From their conservative constraints on both the inclination of the orbits and the mass ratio, the lower limit on the pulsar mass is $1.66 M_{\odot}$, well below the massive pulsars mentioned above. Romani et al. [3] carried out spectroscopic observations of PSR J1311-3430, which is a gamma-ray black-widow pulsar. Their modeling of the quiescent light curve provides constrains on the orbital inclination and mass of the pulsar and its companion. The most simplistic lightcurve fits give $M=2.7 M_{\odot}$. Their multicomponent fits imply a large mass range, however all solutions give pulsar masses in excess of $2.1 M_{\odot}$.

\section{B. Quark phase transition in compact stars, supernovae and gamma ray bursts}

Several scenarios of the quark phase transition in compact stars have been discussed in the literature. They can roughly be grouped into two categories, depending on when the phase transition takes place [15]:

- quark phase transition before deleptonization of the proto-neutron star

- quark phase transition during or after the deleptonization.

In the first case, the phase transition from hadronic to quark matter could already occur in the early postbounce phase of a core-collapse supernova [16-21]. One feature of this mechanism is that it requires a particularly soft EoS, since the formation of a mixed phase of 
quarks and hadrons has to take place at the relatively low densities reached at the moment of core bounce (or immediately after, during the fallback but anyway before deleptonization). Since the densities reached at the moment of the bounce are only moderately dependent on the mass of the progenitor, this mechanism is rather universal, affecting most of the supernovae, although its effect on the explosion can still depend on the mass of the progenitor. In this case, a signal in the emitted neutrinos can be detected [22], associated with the second shock wave which forms when the critical density, separating the mixed phase and the pure quark matter phase, is reached. This neutrino flux would provide a unequivocal signature of a phase transition associated with the SN explosion. Unfortunately, at the moment it seems not possible to reconcile the criterion of a phase transition close to the SN bounce, with a correspondingly soft EoS, with the lower bound on the neutron star maximum mass, which requires a hard EoS at high densities [21].

The second possibility is that the quark phase transition takes places only after, at least partial, deleptonization [23, 24]. In fact, it is well known, that when the pressure due to leptons decreases, the central baryonic density increases and, therefore, hyperon production and the transition to quark matter proceeds easier. A crucial point in this analysis is that the quark phase transition can take place only when strangeness (either in the form of hyperons or kaons) starts being produced in the center of the compact star: at that point, a transition to strange quark matter is possible on the time-scale of the strong interaction [25]. Clearly, a temporal separation can exist in this second scenario, between the moment of core collapse and the moment in which quark matter appears. In this scenario it is possible to have a huge release of energy associated with the combustion of hadronic matter into quark matter [26]. This energy can be the source that powers the central engine of Gamma-Ray Bursts [27, 28]. The time-delay between the moment of the SN explosion and the moment of the quark phase transition could explain a few observed features of Gamma-Ray Bursts, as e.g. the existence of very long quiescent times seen in a few bursts [29] and the possible existence of Gamma-Ray Bursts for which no associated SN explosion is observed [30].

\section{Quark Matter Equation of State}

The equation of state for strongly interacting matter at finite density, i.e. the one that should be extracted from QCD in a dense medium, is a key ingredient for the determination 
of the structure of compact stars. Currently, we are still far from a robust calculation on the lattice in this regime. Nevertheless, one can use extreme limits in baryonic density, where either QCD can be solved in some approximation scheme or nuclear theory models become reliable.

At low densities, below and at the nuclear saturation density, relativistic and nonrelativistic nuclear theory descriptions have achieved a high degree of sophistication and can also be tested by various means. The problem arises in the adjacent region of high densities, where quark matter could also be present. So far, most treatments that include quark matter in compact stars, or are meant to exclude it, still employ the bag model [31 33]. This corresponds to the crudest (yet originally efficient, in the vacuum) way of incorporating confinement, which plainly discards interactions (except from eventual simplified $O\left(\alpha_{s}\right)$ correction). However, after more than a decade of lattice QCD studies at finite temperature and zero density, it became clear that this description is very poor. Besides missing the nature of the phase transition, it also misses the fact that interactions are non-negligible even well above the critical region of deconfinement.

A widely used alternative to bag-model equations of state is the Nambu-Jona-Lasinio (NJL) model, which has been applied to compact stars for about 15 years [34 37]. An attractive feature of this kind of model is that the "bag" pressure (i.e., the pressure difference between perturbative and non-trivial vacuum) is not an input parameter but is dynamically generated via spontaneous chiral-symmetry breaking. This mechanism also leads to effective ("constituent") quark masses, which are (as the bag pressure) density and temperature dependent. The same is true for the pairing gaps in various color-superconducting phases, which can be incorporated naturally [38, 39].

On the other hand, the NJL model is non-renormalizable and therefore its application is restricted to temperatures and chemical potentials well below the cutoff scale. In fact, except for the trivial Stefan-Boltzmann limit in the case without vector interactions, its asymptotic behavior does not agree in general with perturbative QCD. Unfortunately, the standard mean-field approximation does not allow for a realistic description of the hadronic phase either. Although interesting attempts have been made to employ the NJL model beyond mean field in order to construct nuclear matter starting from quark degrees of freedom [40, 41], the results are not yet competitive with state-of-the-art nuclear equations of state. NJL models are therefore mostly used to model the quark-matter equation of state 
above, but not too far above the deconfinement phase transition, where non-perturbative effects (in particular in the strange-quark and color superconducting sector) still play a decisive role. Of course, it is not clear whether model parameters, which have been fitted to vacuum quantities, are still valid in this regime. One may also question the identification of the NJL vacuum pressure with the pressure of the hadronic vacuum, so that an additional bag constant could be introduced [42]. Therefore, while the NJL model can give interesting qualitative insights and uncover new effects, its quantitative predictions should be taken with care.

In that vein, although perturbation theory at finite density should only be reliable at densities much higher than those expected to be found in compact stars, it is a clean framework that contains the effects from interactions and also provides an estimate of its uncertainties in a systematic way, via the dependence on the scale associated with the subtraction point for renormalization. This kind of control is hardly possible in the case of the bag model approach. Moreover, perturbation theory for dense QCD corresponds to the limit any effective treatment should reach at high enough values of the baryonic chemical potential.

The thermodynamic potential for cold QCD was first computed within perturbation theory to $\sim \alpha_{s}^{2}$ decades ago [43-48]. More than ten years ago, corrections $\sim \alpha_{s}^{2}$ with a modern definition of the running coupling constant were used to model the non-ideality in the equation of state for cold, dense QCD with massless quarks [49]. This description also predicted quark stars with masses above $2 M_{\odot}$. (For higher-order computations, see Refs. [50 52]). Remarkably, results from the simple perturbative approach and from treatments that resort to resummation methods and quasiparticle model descriptions [53 57] seem to agree well even for quark chemical potentials $\mu_{q} \sim 1 \mathrm{GeV}$ and smaller.

More recently, it was shown that the inclusion of the running strange quark mass has a non-trivial influence on the equation of state [58] (see also Refs. [59, 60]), where it was argued that effects from both the color superconductivity gap and the strange quark mass should matter in the lower-density sector of the equation of state. Moreover, $\mathcal{O}\left(\alpha_{s}^{2}\right)$ calculations including one massive quark flavor allowing for further non-perturbative effects found that perturbation theory converges reasonably well for quark chemical potentials above $1 \mathrm{GeV}$ 61]. The latter also suggest that quark matter in compact star cores becomes confined to hadrons only slightly above the density of atomic nuclei, and obtain hybrid star masses of up to $M \sim 2 M_{\odot}$, and even larger in the case of strange stars [61, 62]. 


\section{Quark-Hybrid Stars}

Strange quark matter has been hypothesized to exist in self-bound (strange) quark stars, but may also be found in the cores or ordinary "neutron" stars due to the enormously high pressure values at the centers of such objects. The latter are referred to as quark-hybrid stars (see [63] and references therein). These types of stars are comprised of a quark core surrounded by a hadronic shell, which can also contain strangeness via hyperons or a kaon condensate. Usually, the modeling for these stars consists of employing two equations of state, a hadronic and a quark one, switching from one set of degrees of freedom to the other when the equations of state cross. Common choices for the quark EoS are based on the bag model, linear $\sigma$ model, non-linear realization of the $\sigma$ model, NJL model, or Schwinger-Dyson approaches.

The Maxwell and the Gibbs constructions of the phase transition are standard choices. In the second case, a mixed phase of quarks and hadrons appears [64, 65]. Which of the scenarios applies depends strongly on the QCD surface tension, whose value is still highly uncertain. Calculations that include macroscopic structures known as pasta phases show that small surface tensions yield a behavior similar to the Gibbs construction, whereas larger surface tensions reproduce the results of the Maxwell construction [66], reducing the softening of the EoS due to the phase mixing.

A crucial ingredient for obtaining heavy hybrid stars with masses of two $M_{\odot}$ or larger is a stiff equation of state for the quarks. While the simple bag model tends to be too soft to sustain a large core of quark or mixed matter, additional repulsive forces between the quarks prevent a large jump in energy density (latent heat) at the transition [67, 68] and lead to the stabilization of heavy hybrid stars. In this case, complex stars containing nucleons, hyperons and various color superconducting phases are possible [69, 70]. Frequently, reconfinement can occur at high chemical potentials, reverting the system to a hadronic one. This can be avoided by taking into account the finite size of the hadron and including a moderate

excluded volume [71]. We note that an open question remains, however, regarding the discrepancy with lattice results (for the pressure at low baryonic chemical potential) when quark-vector interactions are included [72].

A signature of the existence of hybrid stars could come from a twin star solution, in which two stars (a hadronic and a hybrid one) can exist as stable solutions with similar mass but 
distinctly different radii (see $[71,73,78]$ ). Such scenarios could, in principle, be validated as soon as simultaneous radius and mass measurements become available and they would also point towards a first-order phase transition at small temperatures. A further possible signature of hybrid stars would be a distinct frequency range of gravitational wave emission in the g-mode [79], pulsar backbending [80] and anomalies in the spin-up of neutron stars in low-mass X-ray binaries [81].

In the case that the interiors of high mass compact stars contain quark matter in a crystalline color superconducting state [82 84] or solid quark matter [85] a detectable gravitational wave emission is expected. The characteristic strain of gravitational wave emission predicted by these models could be observed with advanced LIGO. Such a detection can potentially put constraints on the breaking strain and pairing gap of the crystalline color superconducting matter.

Whereas the aforementioned studies assume a first-order transition from hadrons to quarks, also a continuous cross-over is possible [86], similar to the transition at zero chemical potential. This can be done in a unified description of quarks and hadrons and leads to a hybrid star with a continuous increase of the quark fraction without Gibbs construction, generating heavy hybrid stars [71].

Quark matter in high-mass neutron stars has very recently been studied using extensions of the local and non-local Nambu-Jona Lasinio model supplemented with repulsive vector interactions among the quarks (see [87, 88] and references therein). The phase transition from hadronic matter to quark matter has been constructed via the Gibbs condition, which imposes global rather than local electric charge neutrality and baryon number conservation. Depending on the strength of the quark vector repulsion, it was found that an extended mixed phase of hadronic matter and quarks can exist in neutron stars as massive as 2.1 to $2.4 M_{\odot}$. A phase of pure quark matter inside such high-mass neutron stars, while not excluded, is only obtained for certain parametrizations of the underlying Lagrangians. The radii of all these stars are between 12 and $13 \mathrm{~km}$, as expected for neutron stars of that mass.

Finally, at extremely large chemical potentials, not necessarily present inside compact stars, the hadronic or hybrid (containing a mixed phase) EoS must connect with the perturbative QCD EoS. As shown in [61, 62], this is not a simple task, since the perturbative calculation presents error bands and it stops being reliable for baryon chemical potentials of about $1 \mathrm{GeV}$. In addition, so far, we cannot determine in a model-independent fashion if the 
hadronic or hybrid EoS will connect to the pure quark one at low or high densities. A further difficulty arises when chiral symmetry is considered. The restoration of chiral symmetry is an important characteristic of matter at high densities, but it does not necessarily coincide with the critical density for the deconfinement [89, 90]. Such an assumption results, for example, in the quarkyonic matter picture [91, 92].

Recently, an unprecedented fast cooling of the compact star in Cassiopeia A (Cas A) - the youngest known supernova remnant in Milky Way - was inferred from the Chandra observations over a period of ten years [93]. The age of this object is $330 \mathrm{yr}$. The theoretical fits to the cooling behaviour of Cas A can provide important information on the internal composition of compact stars. Hadronic models of compact stars have been invoked to fit the data by assuming a canonical $1.4 M_{\odot}$ mass neutron star. The rapid cooling in these models are mainly due to the pair-breaking process in the hadronic core of the neutron star [94 96]. An alternative model of the cooling of the compact star in Cassiopea A is based on the modeling of the cooling of hybrid stars and accounts for its unusually fast cooling behavior by invoking a phase transition among two different color superconducting phases of quark matter [97]. Specifically, the rapid cooling may be interpreted as an enhancement in the neutrino emission triggered by a transition from a fully gapped, two-flavor, colorsuperconducting phase to a crystalline phase or an alternative gapless, color-superconducting phase. By fine-tuning a single parameter - the temperature of this transition - a specific cooling scenario can be selected that fits the Cas A data. Such a scenario requires a massive $M \sim 2 M_{\odot}$ star and, if correct, should be one of the signals of quark matter in compact stars.

\section{E. Dynamics of Transitions from Nucleonic to Hybrid Stars}

Within the hypothesis of absolutely stable strange quark matter (the Bodmer-Witten hypothesis) there is the possibility that neutron stars convert into quark stars [98]. Once a seed of strange quark matter is formed within hadronic matter, the conversion proceeds as a combustion process where hadronic matter represents the fuel and quark matter the ash. There have been many works on this subject indicating that strong deflagration is the relevant combustion mode for neutron star's burning and that hydrodynamical instabilities play an important role (see for instance [99 101] and references therein). Recent 3+1D hydro- 
dynamic simulations [102, 103] have confirmed that Rayleigh-Taylor instabilities take place, which significantly increase the velocity of conversion: almost the whole star is converted in a time scale of the order of a few milliseconds. The newly born strange star is quite hot, with temperatures of a few tens of $\mathrm{MeV}$ in the center and cools down via neutrino diffusion. For the first time the neutrino signal associated with the conversion has been estimated by use of a diffusion code [103]. Similarly to the signal of proto-neutron stars, a proto-quark star would have an initial luminosity of about $10^{52} \mathrm{erg} / \mathrm{s}$, representing thus a detectable neutrino source if located close enough to our Galaxy.

There are many interesting points that have been raised in the meeting, the most important concerning the full conversion of the star: the burning indeed stops before the whole star is converted, leaving an amount of unburned hadronic material of the order of $0.5 M_{\odot}$. How the conversion proceeds and how long it takes for a full conversion to occur, represent important questions for future studies. It has been also proposed that this result would imply that, even if the Bodmer-Witten hypothesis is correct, only hybrid stars can form. This possibility would rule out the existence of pure quark stars and, possibly, the existence of strangelets in cosmic rays [104]. On the other hand, the existence of pure quark stars would imply that, at least in some cases and for some parametrizations of the equation of state the merger of two strange stars would eject strangelets [105], which in turn would pollute the whole Galaxy potentially triggering the conversion of all neutron stars [106] to strange stars. A better theoretical understanding of the formation of (pure) quark stars is therefore mandatory if the scenario of the existence of two families of compact stars [107], hadronic and quark stars, ought to be viable.

Another interesting question for future studies concerns the possible transition from deflagration to detonation in the burning of a neutron star as proposed for instance in Ref. [108]. At the moment, there are no calculations or numerical simulations which confirm this hypothesis, but this possibility can not be excluded. A detonation would be responsible for a significant amount of matter ejected after the conversion and possible consequences for supernovae and nucleosynthesis have been analysed (see Ref. [109]).

Another scenario leading to the transformation of a neutron star into a hybrid star was also discussed during the meeting. It could be realized if the phase transition from the hadronic to the quark phase is strong enough, so that the jump in relative densities exceeds a critical value of about $1.5[110-112]$. If in the course of the evolution (by cooling, slowing 
down, increasing the mass due to accretion) the central density reaches this critical value, the star "rolls down" into a new equilibrium state, where the quark core occupies a significant fraction of the star. Up to now only very schematic calculations of this process have been

made, see [113, 114]. They show that the core radius first grows exponentially with a characteristic time of a few milliseconds and then experiences damped oscillations around a new equilibrium state. As estimated in Refs. [115, 116], the energy released in this transition could be as large as $10^{52} \mathrm{erg}$. It should reheat the star and induce an additional neutrino burst. Certainly, more realistic simulations of such a catastrophic process could be done using modern computational resources.

\section{F. Signals for Quark Matter in Compact Stars}

In addition to the signals mentioned above, strange quark stars or the presence of a new form of matter in the core of compact stars can lead to the following astrophysical signatures.

\section{Dressed strange quark stars and Eddington Limit}

A bare quark star differs qualitatively from a neutron star which has a density at the surface of about 0.1 to $1 \mathrm{~g} / \mathrm{cm}^{3}$. The thickness of the quark surface is just about $1 \mathrm{fm}$, the length scale of the strong interaction. The electrons at the surface of a quark star are held to quark matter electrostatically, and the thickness of the electron surface is several hundred fermis. Since neither component, electrons and quark matter, are held in place gravitationally, the Eddington limit to the luminosity that a static surface may emit does not apply, so that bare quark stars may have photon luminosities much greater than $10^{38} \mathrm{erg} / \mathrm{s}$. It has been shown in [117] that this value may be exceeded by many orders of magnitude by the luminosity of $e^{+} e^{-}$pairs produced by the Coulomb barrier at the surface of a hot strange star. For a surface temperature of $\sim 10^{11} \mathrm{~K}$, the luminosity in the outflowing pair plasma was calculated to be as high as $\sim 3 \times 10^{51} \mathrm{erg} / \mathrm{s}$. Such an effect may be a good observational signature of bare strange stars [117-120]. If the strange star is dressed, that is, enveloped in a nuclear crust, however, the surface made of ordinary atomic matter would be subject to the Eddington limit. Hence the photon emissivity of a dressed quark star would be the same as for an ordinary neutron star. But note that if quark matter at the stellar 
surface is in the CFL (Color-Flavor Locked) phase, the process of $e^{+} e^{-}$pair creation at the stellar quark matter surface may be turned off. This may be different for the early stages of a very hot CFL quark star [121].

\section{Mass-Radius relationship and rapid rotation of quark stars}

In contrast to neutron stars, the radii of self-bound quark stars decrease the lighter the stars, according to $M \propto R^{3}$. The existence of nuclear crusts on quark stars changes the situation drastically [63, 122]. Since the crust is bound gravitationally, the mass-radius relationship of quark stars with crusts is qualitatively similar to neutron stars. In general, quark stars with or without nuclear crusts possess smaller radii than neutron stars. This implies that quark stars have smaller mass shedding (break-up) periods than neutron stars. Moreover, due to the smaller radii of quarks stars, the complete sequence of quark stars and not just those close to the mass peak, as it is the case for neutron stars - can sustain extremely rapid rotation [63, 122]. In particular, a strange star with a typical pulsar mass of around $1.45 M_{\odot}$ has a Kepler period in the approximate range of $0.55 \lesssim P_{\mathrm{K}} / \mathrm{msec} \lesssim 0.8$ [122, 123]. This is to be compared with $P_{\mathrm{K}} \sim 1 \mathrm{msec}$ for neutron stars of the same mass.

Another novelty of the strange quark matter hypothesis concerns the existence of a new class of white-dwarf-like objects, referred to as strange (quark matter) dwarfs [122]. The mass-radius relationship of the latter differs somewhat from the mass-radius relationship of ordinary white dwarfs, which may be testable observationally in the future. Until recently, only rather vague tests of the theoretical mass-radius relationship of white dwarfs were possible. This has changed dramatically because of the availability of new data emerging from the Hipparcos project [124]. These data allow the first accurate measurements of white dwarf distances and, as a result, establishing the mass-radius relation of such objects empirically.

\section{Features of electrically charged strange quark stars}

One of the most amazing features of strange quark stars concerns the existence of ultrahigh electric fields on their surfaces, which, for ordinary (i.e., non-superconducting) quark matter, is around $10^{18} \mathrm{~V} / \mathrm{cm}$. If strange matter forms a color superconductor, as expected for 
such matter, the strength of the electric field may increase to values that exceed $10^{19} \mathrm{~V} / \mathrm{cm}$. The energy density associated with such huge electric fields is on the same order of magnitude as the energy density of strange matter itself, which alters the masses and radii of strange quark stars at the $15 \%$ and $5 \%$ level, respectively $[125]$. Similar effects are predicted also for hybrid stars with a small net electric charge [126]. Such mass increases facilitate the interpretation of massive compact stars, with masses of around $2 M_{\odot}$, as possible strange quark stars.

The electrons at the surface of a quark star are not necessarily in a fixed position but may rotate with respect to the quark matter star [127]. In this event magnetic fields can be generated which, for moderate effective rotational frequencies between the electron layer and the stellar body, agree with the magnetic fields inferred for several Central Compact Objects (CCOs). These objects could thus be interpreted as quark stars whose electron atmospheres rotate at frequencies that are moderately different $(\sim 10 \mathrm{~Hz})$ from the rotational frequency of the quark star itself.

Last but not least, we mention that the electron surface layer may be strongly affected by the magnetic field of a quark star in such a way that the electron layer performs vortex hydrodynamical oscillations [128]. The frequency spectrum of these oscillations has been derived in analytic form in [128]. If the thermal X-ray spectra of quark stars are modulated by vortex hydrodynamical oscillations, the thermal spectra of compact stars, foremost central compact objects (CCOs) and X-ray dim isolated neutron stars (XDINSs), could be used to verify the existence of these vibrational modes observationally. The central compact object 1E 1207.4-5209 appears particularly interesting in this context, since its absorption features at $0.7 \mathrm{keV}$ and $1.4 \mathrm{keV}$ can be comfortably explained in the framework of the hydro-cyclotron oscillation model [128].

\section{Possible connection of quark stars to SGRs, AXPs, and XDINs}

Rotating superconducting quark stars ought to be threaded with rotational vortex lines, within which the star's interior magnetic field is at least partially confined. The vortices (and thus magnetic flux) would be expelled from the star during stellar spin-down, leading to magnetic re-connection at the surface of the star and the prolific production of thermal energy. In has been shown in [129] that this energy release can re-heat quark stars 
to exceptionally high temperatures, such as observed for Soft Gamma Repeaters (SGRs), Anomalous X-Ray pulsars (AXPs), and X-ray dim isolated neutron stars (XDINs), and that SGRs, AXPs, and XDINs may be linked ancestrally [129].

\section{Rotation-driven compositional changes}

The change in central density of a neutron star whose frequency varies from zero to the mass shedding (Kepler) frequency can be as large as 50 to 60\% [63]. This suggests that changes in the rotation rate of a neutron star may drive phase transitions and/or lead to significant compositional changes in the star's core [63, 96, 130]. As a case in point, for some rotating neutron stars the mass and initial rotational frequency may be just such that the central density rises from below to above the critical density for dissolution of baryons into their quark constituents. This may be accompanied by a sudden shrinkage of the neutron star, effecting the star's moment of inertia and, thus, its spin-down behavior. As shown in [80], the spin-down of such a neutron star may be stopped or even reversed for tens of thousands to hundreds of thousands of years [63, 80]. The observation of an isolated neutron star which is spinning-up, rather than down, could thus hint at the existence of quark matter in its core.

\section{Quark-hadron Coulomb lattice (electron Bremsstrahlung)}

Because of the competition between the Coulomb and the surface energies associated with the positively charged regions of nuclear matter and negatively charged regions of quark matter, the mixed phase may develop geometrical structures (e.g., blobs, rods, slabs), similarly to what is expected of the sub-nuclear liquid-gas phase transition [64]. The consequences of such a Coulomb lattice for the thermal and transport properties of neutron stars have been studied in [65]. It was found that at low temperatures of $T \lesssim 10^{8} \mathrm{~K}$ the neutrino emissivity from electron-blob Bremsstrahlung scattering is at least as important as the total contribution from all other Bremsstrahlung processes (such as nucleon-nucleon and quark-quark Bremsstrahlung) and modified nucleon and quark Urca processes. It is also worth noting that the scattering of degenerate electrons off rare phase blobs in the mixed phase region lowers the thermal conductivity by several orders of magnitude compared to 
a quark-hadron phase without geometric patterns. This may lead to significant changes in the thermal evolution of the neutron stars containing solid quark-hadron cores, which has not yet been studied.

\section{Pycnonuclear reaction rates}

The presence of strange quark nuggets in the crustal matter of neutron stars could be a consequence of Witten's strange quark matter hypothesis. The impact of such nuggets on the pycnonuclear reaction rates among heavy atomic nuclei has been studied in [131]. Particular emphasis was put on the consequences of color superconductivity on the reaction rates. Depending on whether or not quark nuggets are in a color superconducting state, their electric charge distributions differ drastically, which was found to have dramatic consequences for the pycnonuclear reaction rates in the crusts of neutron stars. Future nuclear fusion network calculations may thus have the potential to shed light on the existence of strange quark matter nuggets and on whether they are in a color superconducting state, as suggested by QCD.

\section{8. $\quad$-mode instability}

The r-mode instability of a rotating neutron star dissipates the star's rotational energy by coupling the angular momentum of the star to gravitational waves [132 134]. This instability can be active in a newly formed isolated neutron star as well as in old neutron stars being spun up by accretion of matter from binary stars. If the interior contains quark matter, the r-mode instability and the gravitational wave signal may carry information about quark matter [135 139$]$.

\section{Quark Novae}

The conversion of a neutron star to a hypothetical quark star could lead to quark novae. Such events could explain gamma ray bursts [140], the production of heavy elements such as platinum through r-process nucleosynthesis [141], and double-humped super-luminous supernovae [142]. 


\section{RELATION TO OTHER FIELDS}

\section{A. Hyperons in Compact Stars}

A previous task force meeting called HyperoNS2012 was held November 21-24, 2012 at the Nicolaus Copernicus Astronomical Center (CAMK), in Warsaw, Poland. The scientific program of the meeting was prepared by Pawel Haensel (CAMK) and Jürgen SchaffnerBielich (then Heidelberg University). The motivation for this task force meeting was clear: the measurement of a $2 M_{\odot}$ neutron star [1] forced us, dense matter theorists, to reconsider the problem of the structure of massive neutron star cores. In particular, the problem of too strong a softening of the equation of state of dense baryonic matter due to the presence of hyperons at high density, inconsistent with such a high neutron star mass, reappeared in a very dramatic way. Two basic "solutions" for this puzzle were proposed: (a) additional repulsion between hyperons due to a vector meson coupled to hyperons only; (b) a transition to a very stiff quark plasma before the hyperon threshold. Note, that both "solutions" have their own problems and difficulties.

It seemed timely and useful to organize, two years after the $2 M_{\odot}$ neutron star announcement, a three-day task force meeting devoted to the present status of possible existence of hyperon/quark cores in neutron star. The knowledge of the hyperon interactions was

reviewed. Proponents of different solutions to the "hyperon puzzle" had the opportunity to present their arguments, which were then critically discussed by the participants of the meeting.

Marcello Baldo (Catania) presented a review of the modern many-body theory of nuclear matter, and its relation to the "hyperon puzzle". A non-linear relativistic mean-field (RMF) model yielding neutron star with hyperon cores and $M_{\max }>2 M_{\odot}$ was presented by Ilona Bednarek (Katowice). David Blaschke (Wroclaw) gave an overview of quark-matter theories in the context of hybrid stars. Interesting new results on the appearance of strangeness in neutron star cores were presented by Micaela Oertel (Meudon). The effect of the symmetry energy of nuclear matter on the strangeness content of neutron star cores was discussed by Constanca Providencia (Coimbra). Jürgen Schaffner-Bielich (Heidelberg) gave an overview of the antikaon properties in dense matter. Various approaches to the properties of hybrid stars, and perspectives for a unified formulation of the description of hadron and quark 
phases were described by Stefan Schramm (Frankfurt). Nonrelativistic Brueckner-HartreeFock calculations of hypernuclear matter with Nijmegen NY potentials were discussed and their implications for hypernuclei and neutron star were critically examined by Hans-Josef Schulze (Catania). Armen Sedrakian (Frankfurt) discussed the structure of color superconducting cores of hybrid stars, and the cooling of such stars, and confronted theoretical models with recent observations of cooling of the pulsar in Cas A. A hybrid model that combines an extended RMF description of hyperonic matter and a modified Nambu-Jona-Lasinio model for quark matter applied to hybrid stars was presented by Stefan Typel (Darmstadt). Isaac Vidana (Coimbra) presented recent results on the stiffening effect of three-body forces on the EoS of hypernuclear matter. He showed that the three-body forces are unable to make the EoS of hyperonic matter sufficiently stiff to yield $M_{\max }>2 M_{\odot}$. Constraints imposed on the phase transition to quark matter in neutron star cores, implied by the $M_{\max }>2 M_{\odot}$ condition, were presented by Pawel Haensel (Warsaw).

The talks were followed by lively and inspiring discussion. The two solar mass limit is found to be a challenge for hyperonic cores in many-body theories, and a strong tuning following a selection of a RMF model, is required to find "a way out"; Brueckner-type theories are unable to yield $M_{\max }>2 M_{\odot}$. Quark cores replacing a hyperon core have to be very stiff and the hadron-quark matter phase transition should be associated with a small density jump very different from the twin star condition. Several projects were inspired in these discussions and reported a year later at the EMMI Rapid Reaction Task Meeting in Frankfurt in October of 2013. In this way the Warsaw task force initiated a series of meetings on current problems in the description of compact stars and dense matter physics.

There were 22 participants in the Warsaw meeting. All living and travel expenses of non-Polish participants (15) were covered by the CAMK from the COMPSTAR-POL Polish grant. More details, including talks from the "HyperoNS2012" meeting can be found at http://www.camk.edu.pl/conf/Hyperons2012.

The hyperon puzzle was also a topic at the task force meeting reported here during the discussions as it is important to fix the low to medium density equation of state for hybrid stars. More information on the hyperon-hyperon interactions is needed to address the issue in more detail but might be available in the near future. Two particle correlations of hyperons emitted in relativistic heavy-ion collisions might come from experiments at CERN's LHC. Recent lattice simulations of the binding energy of dibaryons with hyperons have already 
demonstrated that bound dibaryon states with hyperons might exist. These lattice results are encouraging but are only calculated for unphysical (heavy) pion masses and need to be extrapolated to the physical limit.

\section{B. Messages from Heavy-Ion Physics}

Heavy-ion collisions at intermediate energies bring important information about properties of compressed baryonic matter. Using ions with different $\mathrm{Z} / \mathrm{N}$ ratios opens the possibility to study isospin asymmetric matter. As calculations show, see e.g. Ref. [143], baryon densities up to $(2-4) \rho_{0}$ can be reached at intermediate stages of heavy-ion collisions at beam energies of $0.2-2.0 \mathrm{AGeV}$. This density interval spans a significant fraction of the interior of compact stars. Such experiments allow to obtain valuable information about the densitydependence of the symmetry energy and the composition of isospin-asymmetric matter. Recently, the old FOPI/LAND data on elliptic flow of neutrons and light charged particle production in $\mathrm{Au}+\mathrm{Au}$ collisions at $0.4 \mathrm{AGeV}$ [144] were reanalyzed to extract the exponent $\gamma$ in the density dependence of the symmetry energy $\left(\rho / \rho_{0}\right)^{\gamma}$ : comparison of these data with predictions of two Quantum Molecular Dynamics models [145, 146] has shown that $\gamma=0.9 \pm 0.4$. The analysis of various sets of nuclear data, sensitive to the density dependence of symmetry energy, has allowed to extract the value of its first derivative at $\rho \approx \rho_{0}$, expressed as $L /\left(3 \rho_{0}\right)$. The parameter $L$ is found to lie in the range of about $40-60 \mathrm{MeV}$ [147].

The analysis of the subthreshold production of kaons in heavy-ion collisions measured by the KaoS collaboration [148] revealed that the bulk nuclear equation of state around $(2-3) \rho_{0}$ is soft within transport simulations 149, 150]. Implications for the maximum mass of neutron stars have been addressed in [151] showing that the equation of state extracted from these analysis is compatible with the new pulsar mass constraint if the equation of state stiffens considerably at higher density. Neutron star masses of more than $2.7 M_{\odot}$ could not be reached in [151] when enforcing causality by using the stiffest possible equation of state and the constraint from the KaoS data analysis.

Presently, it is well established experimentally that strange hadrons are abundantly produced in heavy-ion collisions at intermediate energies. Their observed abundances closely follow the predictions of simple thermal models, see e.g. [152, 153]. In particular, the ratio 
of Lambda and proton yields, $\Lambda / p$, at freeze-out is about 1 at low SPS energies. Transport calculations show that strangeness (and anti-strangeness) is mostly produced at the initial stage of the collision and then decreases slowly towards freeze-out [154]. Thus, one should conclude that the abundance of strange baryons in the dense baryonic matter produced in such collisions is comparable to the abundance of nucleons. No signs of hyperon suppression have been found so far. Therefore, there are no reasons to ignore the presence of hyperons in dense baryonic matter, as done in some works. The participants of the task force meeting have agreed that the models using entirely nucleonic degrees of freedom up to $(4-5) \rho_{0}$ and ignoring hyperons should be considered as "exotic scenarios", even if they satisfy the mass-radius constraints on compact stars.

\section{OUTLOOK}

The need for a simultaneous measurement of the mass and the radius to say a few percent precision seems to be crucial to learn more about the properties of high-density matter in the cores of compact stars. Arguments are put forward during the discussions that exotic matter inside neutron stars implies radii of more than $11 \mathrm{~km}$ for a $1.4 M_{\odot}$ neutron star assuming a speed of sound similar to the one present in the MIT bag model and a maximum mass of $2 M_{\odot}$ (see figure 5 of ref. [155]). As it became clear during the meeting, the simple MIT bag model is ruled out from lattice data, so that the arguments have to be taken with great caution. The limit on the radius was shown to be beaten by more advanced models as presented during the task force meeting, see e.g. [156], so that radii of less than $11 \mathrm{~km}$ are possible with strange matter cores. Still, the issue is not fully addressed and needs to be reconsidered with improved models for the high-density equation of state.

It is promising that a new technique has been developed in order to extract masses and radii of compact stars: the study of the X-ray flux modulations due to rotating hot spots on the surface of neutron stars. Taking into account general relativity corrections, the farside spot becomes more visible for smaller stars through gravitational light-bending, which depends on the compactness $M / R$ of the compact star. This technique can be applied to study thermal pulsation of nearby millisecond pulsars and burst oscillations from low-mass X-ray binaries. Both the approved NICER mission of NASA [9] and the proposed LOFT

satellite [10] are adopting this technique. Error bars smaller than $\sim 10 \%$ are expected for the 
radius measurement, what would finally put rather tight constraints on the dense matter EoS.

Furthermore, the study of the high-density equation of state for compact stars seems to be at a stage where the old models of QCD need to be abandoned. It emerged from the task force that the simple MIT bag model is excluded from observations and from lattice data. Vector interactions stiffen the quark matter equation of state but are in variance with asymptotic freedom and lattice data. Improved models of QCD are available but need to be extended for astrophysical applications to achieve progress in further studies. Indeed, the task force meeting succeeded in achieving this task by initiating joint work where perturbative QCD calculations at high baryon densities were used to describe the equation of state suitable for compact stars [157, 158]. Judging from the lively atmosphere created at the task force meeting, more joint work in this direction will surely follow.

\section{ACKNOWLEDGMENTS}

This work was supported by the Alliance Program of the Helmholtz Association (HA216/EMMI). We thank all the participants for the lively discussions and the constructive atmosphere generated at this meeting.

[1] P. Demorest, T. Pennucci, S. Ransom, M. Roberts, and J. Hessels Nature 467 (2010) 1081-1083, arXiv:1010.5788 [astro-ph.HE].

[2] J. Antoniadis, P. C. Freire, N. Wex, T. M. Tauris, R. S. Lynch, et al. Science 340 (2013) 6131, arXiv:1304.6875 [astro-ph.HE].

[3] R. W. Romani, A. V. Filippenko, J. M. Silverman, S. B. Cenko, J. Greiner, A. Rau, J. Elliott, and H. J. Pletsch Astrophys. J. Lett. 760 (2012) L36, arXiv:1210.6884 [astro-ph.HE],

[4] D. L. Kaplan, V. B. Bhalerao, M. H. van Kerkwijk, D. Koester, S. R. Kulkarni, et al. Astrophys.J. 765 (2013) 158, arXiv:1302.2492 [astro-ph.SR].

[5] R. Smits, D. R. Lorimer, M. Kramer, R. Manchester, B. Stappers, C. J. Jin, R. D. Nan, and D. Li Astron. Astrophys. 505 (2009) 919-926, arXiv:0908.1689 [astro-ph.IM]. 
[6] J. Cordes, M. Kramer, T. Lazio, B. Stappers, D. Backer, et al. New Astron.Rev. 48 (2004) 1413, arXiv:astro-ph/0505555 [astro-ph].

[7] LIGO Scientific and Virgo Collaboration, J. Abadie et al. Class.Quant.Grav. 27 (2010) 173001, arXiv:1003.2480 [astro-ph.HE].

[8] LIGO and Virgo Collaboration, J. Aasi et al. Phys.Rev. D88 (2013) 062001,

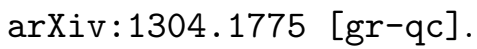

[9] K. C. Gendreau et al. Proc. SPIE Space Telescopes and Instrumentation 8443 (2012) 844313.

[10] M. Feroci et al. Proc. SPIE Space Telescopes and Instrumentation 8443 (2012) 84432D.

[11] E. Bozzo, J. W. den Herder, M. Feroci, and L. Stella, "LOFT - Large area Observatory For X-ray Timing," in Extreme and Variable High Energy Sky (Extremesky 2011). 2011. arXiv:1112.5473 [astro-ph.IM].

[12] E. Bozzo, L. Stella, M. van der Klis, A. Watts, D. Barret, et al. arXiv:1312.1697 [astro-ph.IM].

[13] S. Bogdanov Astrophys.J. 762 (2013) 96, arXiv:1211.6113 [astro-ph.HE].

[14] M. van Kerkwijk, R. Breton, and S. Kulkarni Astrophys.J. 728 (2011) 95, arXiv:1009.5427 [astro-ph.HE].

[15] A. Drago, G. Pagliara, G. Pagliaroli, F. L. Villante, and F. Vissani AIP Conf.Proc. 1056 (2008) 256-263, arXiv:0809.0518 [astro-ph].

[16] N. A. Gentile, M. B. Aufderheide, G. J. Mathews, F. D. Swesty, and G. M. Fuller Astrophys. J. 414 (1993) 701-711.

[17] A. Drago and U. Tambini J. Phys. G25 (1999) 971-979, arXiv:astro-ph/9703138.

[18] K. Nakazato, K. Sumiyoshi, and S. Yamada Phys. Rev. D77 (2008) 103006, arXiv:0804.0661 [astro-ph].

[19] I. Sagert, M. Hempel, G. Pagliara, J. Schaffner-Bielich, T. Fischer, A. Mezzacappa, F.-K. Thielemann, and M. Liebendörfer Phys. Rev. Lett. 102 (2009) 081101, arXiv:0809.4225 [astro-ph].

[20] T. Fischer, I. Sagert, G. Pagliara, M. Hempel, J. Schaffner-Bielich, et al. Astrophys.J.Suppl. 194 (2011) 39, arXiv:1011.3409 [astro-ph.HE],

[21] I. Sagert, T. Fischer, M. Hempel, G. Pagliara, J. Schaffner-Bielich, et al. Acta Phys.Polon. B43 (2012) 741-748, arXiv:1112.6328 [astro-ph.HE], 
[22] B. Dasgupta, T. Fischer, S. Horiuchi, M. Liebendörfer, A. Mirizzi, I. Sagert, and J. Schaffner-Bielich Phys. Rev. D81 (2010) 103005, arXiv:0912.2568 [astro-ph.HE].

[23] J. A. Pons, A. W. Steiner, M. Prakash, and J. M. Lattimer Phys. Rev. Lett. 86 (2001) 5223-5226, astro-ph/0102015.

[24] D. Aguilera, D. Blaschke, and H. Grigorian Astron.Astrophys. 416 (2004) 991-996, arXiv:astro-ph/0212237 [astro-ph].

[25] K. Iida and K. Sato Phys.Rev. C58 (1998) 2538-2559, arXiv:nucl-th/9808056 [nucl-th].

[26] I. Bombaci and B. Datta Astrophys.J. 530 (2000) L69, arXiv:astro-ph/0001478 [astro-ph].

[27] Z. Berezhiani, I. Bombaci, A. Drago, F. Frontera, and A. Lavagno Astrophys.J. 586 (2003) 1250-1253, arXiv:astro-ph/0209257 [astro-ph].

[28] A. Drago, A. Lavagno, and G. Pagliara Phys.Rev. D69 (2004) 057505, arXiv:nucl-th/0401052 [nucl-th].

[29] A. Drago and G. Pagliara Astrophys. J. 665 (2007) 1227-1234, astro-ph/0512602.

[30] C. D. Dermer Astrophys.J. 684 (2008) 430, arXiv:astro-ph/0703223 [ASTRO-PH].

[31] E. Witten Phys. Rev. D 30 (1984) 272.

[32] C. Alcock, E. Farhi, and A. Olinto Astrophys. J. 310 (1986) 261.

[33] P. Haensel, J. L. Zdunik, and R. Schaeffer Astron. Astrophys. 160 (1986) 121.

[34] K. Schertler, S. Leupold, and J. Schaffner-Bielich Phys. Rev. C 60 (1999) 025801, arXiv:astro-ph/9901152.

[35] M. Baldo, M. Buballa, F. Burgio, F. Neumann, M. Oertel, et al. Phys.Lett. B562 (2003) 153-160, arXiv:nucl-th/0212096 [nucl-th].

[36] M. Buballa, F. Neumann, M. Oertel, and I. Shovkovy Phys. Lett. B 595 (2004) 36-43, nucl-th/0312078.

[37] T. Klähn, D. Blaschke, F. Sandin, C. Fuchs, A. Faessler, H. Grigorian, G. Röpke, and J. Trümper Phys. Lett. B654 (2007) 170-176, arXiv:nucl-th/0609067.

[38] M. Buballa Phys.Rept. 407 (2005) 205-376, arXiv:hep-ph/0402234 [hep-ph].

[39] R. Anglani, R. Casalbuoni, M. Ciminale, R. Gatto, N. Ippolito, et al. arXiv:1302.4264 [hep-ph]. 
[40] S. Lawley, W. Bentz, and A. W. Thomas J.Phys. G32 (2006) 667-680, arXiv:nucl-th/0602014 [nucl-th].

[41] J.-c. Wang, Q. Wang, and D. H. Rischke Phys.Lett. B704 (2011) 347-353, arXiv:1008.4029 [nucl-th].

[42] G. Pagliara and J. Schaffner-Bielich Phys. Rev. D 77 (2008) 063004, arXiv:0711.1119 [astro-ph].

[43] B. A. Freedman and L. D. McLerran Phys.Rev. D16 (1977) 1130.

[44] B. A. Freedman and L. D. McLerran Phys.Rev. D16 (1977) 1147.

[45] B. A. Freedman and L. D. McLerran Phys.Rev. D16 (1977) 1169.

[46] B. Freedman and L. McLerran Phys. Rev. D 17 (1978) 1109.

[47] V. Baluni Phys. Rev. D 17 (1978) 2092.

[48] T. Toimela Int.J.Theor.Phys. 24 (1985) 901.

[49] E. S. Fraga, R. D. Pisarski, and J. Schaffner-Bielich Phys. Rev. D 63 (2001) 121702(R), hep-ph/0101143.

[50] A. Vuorinen Phys.Rev. D68 (2003) 054017, arXiv:hep-ph/0305183 [hep-ph].

[51] A. Ipp, A. Rebhan, and A. Vuorinen Phys.Rev. D69 (2004) 077901, arXiv:hep-ph/0311200 [hep-ph].

[52] A. Ipp, K. Kajantie, A. Rebhan, and A. Vuorinen Phys.Rev. D74 (2006) 045016. arXiv:hep-ph/0604060 [hep-ph].

[53] A. Peshier, B. Kämpfer, and G. Soff Phys.Rev. C61 (2000) 045203, arXiv:hep-ph/9911474 [hep-ph].

[54] A. Peshier, B. Kämpfer, and G. Soff Phys.Rev. D66 (2002) 094003, arXiv:hep-ph/0206229 [hep-ph].

[55] J. Blaizot, E. Iancu, and A. Rebhan Phys.Rev. D63 (2001) 065003, arXiv:hep-ph/0005003 [hep-ph].

[56] J. O. Andersen and M. Strickland Phys. Rev. D 66 (2002) 105001, hep-ph/0206196.

[57] A. Rebhan and P. Romatschke Phys.Rev. D68 (2003) 025022 , arXiv:hep-ph/0304294 [hep-ph].

[58] E. S. Fraga and P. Romatschke Phys.Rev. D71 (2005) 105014, arXiv:hep-ph/0412298 [hep-ph],

[59] M. Alford and S. Reddy Phys. Rev. D 67 (2003) 074024, nucl-th/0211046. 
[60] M. Alford, M. Braby, M. Paris, and S. Reddy Astrophys.J. 629 (2005) 969-978, arXiv:nucl-th/0411016 [nucl-th].

[61] A. Kurkela, P. Romatschke, and A. Vuorinen Phys. Rev. D81 (2010) 105021, arXiv:0912.1856 [hep-ph].

[62] A. Kurkela, P. Romatschke, A. Vuorinen, and B. Wu ArXiv e-prints (2010), arXiv:1006.4062 [astro-ph.HE].

[63] F. Weber Prog. Part. Nucl. Phys. 54 (2005) 193-288, astro-ph/0407155.

[64] N. K. Glendenning Phys.Rev. D46 (1992) 1274-1287.

[65] X. Na, R. Xu, F. Weber, and R. Negreiros Phys.Rev. D86 (2012) 123016, arXiv:1208.5022 [astro-ph.SR].

[66] N. Yasutake, T. Noda, H. Sotani, T. Maruyama, and T. Tatsumi arXiv:1208.0427 [astro-ph.HE].

[67] J. Coelho, C. Lenzi, M. Malheiro, J. Marinho, R.M., and M. Fiolhais Int.J.Mod.Phys. D19 (2010) 1521-1524, arXiv:1001.1661 [nucl-th].

[68] C. Lenzi and G. Lugones Astrophys.J. 759 (2012) 57, arXiv:1206.4108 [astro-ph.SR],

[69] L. Bonanno and A. Sedrakian Astron.Astrophys. 539 (2012) A16, arXiv:1108.0559 [astro-ph.SR].

[70] S. Weissenborn, I. Sagert, G. Pagliara, M. Hempel, and J. Schaffner-Bielich Astrophys.J. 740 (2011) L14, arXiv:1102.2869 [astro-ph.HE].

[71] S. Schramm, V. Dexheimer, R. Negreiros, J. Steinheimer, and T. Schürhoff arXiv:1310.5804 [astro-ph.SR].

[72] J. Steinheimer and S. Schramm Phys.Lett. B696 (2011) 257-261, arXiv:1005.1176 [hep-ph].

[73] U. H. Gerlach Phys.Rev. 172 (1968) 1325-1330.

[74] B. Kämpfer J.Phys. A14 (1981) L471-L475.

[75] N. K. Glendenning and C. Kettner Astron. Astrophys. 353 (2000) L9, astro-ph/9807155.

[76] K. Schertler, C. Greiner, J. Schaffner-Bielich, and M. H. Thoma Nucl. Phys. A677 (2000) 463, astro-ph/0001467.

[77] D. Alvarez-Castillo and D. Blaschke arXiv:1304.7758 [astro-ph.HE].

[78] R. Negreiros, V. Dexheimer, and S. Schramm Phys.Rev. C85 (2012) 035805, arXiv:1011.2233 [astro-ph.HE]. 
[79] C. Flores and G. Lugones arXiv:1310.0554 [astro-ph.HE].

[80] N. K. Glendenning, S. Pei, and F. Weber Phys. Rev. Lett. 79 (1997) 1603.

[81] N. K. Glendenning and F. Weber Astrophys.J. 559 (2001) L119, arXiv:astro-ph/0003426 [astro-ph].

[82] L.-M. Lin Phys.Rev. D76 (2007) 081502, arXiv:0708.2965 [astro-ph].

[83] B. Knippel and A. Sedrakian Phys.Rev. D79 (2009) 083007, arXiv:0901.4637 [astro-ph.SR].

[84] N. K. Johnson-McDaniel and B. J. Owen Phys.Rev. D88 (2013) 044004, arXiv:1208.5227 [astro-ph.SR].

[85] R.-X. Xu Astrophys.J. 596 (2003) L59-L62, arXiv:astro-ph/0302165 [astro-ph].

[86] N. M. Bratovic, T. Hatsuda, and W. Weise Phys.Lett. B719 (2013) 131-135, arXiv:1204.3788 [hep-ph].

[87] M. Orsaria, H. Rodrigues, F. Weber, and G. Contrera Phys.Rev. D87 (2013) 023001, arXiv:1212.4213 [astro-ph.SR].

[88] M. Orsaria, H. Rodrigues, F. Weber, and G. Contrera Phys.Rev. C89 (2014) 015806 arXiv:1308.1657 [nucl-th].

[89] S. Borsanyi et al. JHEP 1009 (2010) 073, arXiv:1005.3508 [hep-lat].

[90] A. Bazavov, T. Bhattacharya, M. Cheng, C. DeTar, H. Ding, et al. Phys.Rev. D85 (2012) 054503, arXiv:1111.1710 [hep-lat].

[91] L. McLerran and R. D. Pisarski Nucl. Phys. A796 (2007) 83-100, arXiv:0706.2191 [hep-ph].

[92] A. Andronic, D. Blaschke, P. Braun-Munzinger, J. Cleymans, K. Fukushima, et al. Nucl.Phys. A837 (2010) 65-86, arXiv:0911.4806 [hep-ph].

[93] K. Elshamouty, C. Heinke, G. Sivakoff, W. Ho, P. Shternin, et al. Astrophys.J. 777 (2013) 22, arXiv:1306.3387 [astro-ph.HE].

[94] D. Page, M. Prakash, J. M. Lattimer, and A. W. Steiner Phys.Rev.Lett. 106 (2011) 081101, arXiv:1011.6142 [astro-ph.HE].

[95] D. Blaschke, H. Grigorian, D. Voskresensky, and F. Weber Phys.Rev. C85 (2012) 022802 , arXiv:1108.4125 [nucl-th].

[96] R. Negreiros, S. Schramm, and F. Weber Phys.Lett. B718 (2013) 1176-1180, arXiv:1103.3870 [astro-ph.HE]. 
[97] A. Sedrakian Astron.Astrophys. 555 (2013) L10, arXiv:1303.5380 [astro-ph.HE].

[98] N. Itoh Prog.Theor.Phys. 44 (1970) 291.

[99] J. Horvath and O. Benvenuto Phys.Lett. B213 (1988) 516-520.

[100] G. Lugones, O. Benvenuto, and H. Vucetich Phys.Rev. D50 (1994) 6100-6109.

[101] A. Drago, A. Lavagno, and I. Parenti Astrophys.J. 659 (2007) 1519-1535, arXiv:astro-ph/0512652 [astro-ph].

[102] M. Herzog and F. K. Ropke Phys.Rev. D84 (2011) 083002 , arXiv:1109.0539 [astro-ph.HE].

[103] G. Pagliara, M. Herzog, and F. Ropke Phys.Rev. D87 (2013) 103007, arXiv:1304.6884 [astro-ph.HE].

[104] J. Madsen Phys.Rev. D71 (2005) 014026, arXiv:astro-ph/0411538 [astro-ph].

[105] A. Bauswein, H.-T. Janka, R. Oechslin, G. Pagliara, I. Sagert, J. Schaffner-Bielich, M. Hohle, and R. Neuhäuser Phys. Rev. Lett. 103 (2009) 011101, arXiv:0812.4248 [astro-ph].

[106] G. A. Medina-Tanco and J. Horvath Astrophys.J. 464 (1996) 354-358.

[107] A. Drago, A. Lavagno, and G. Pagliara arXiv:1309.7263 [nucl-th].

[108] B. Niebergal, R. Ouyed, and P. Jaikumar Phys.Rev. C82 (2010) 062801, arXiv:1008.4806 [nucl-th].

[109] R. Ouyed, B. Niebergal, and P. Jaikumar arXiv:1304.8048 [astro-ph.HE].

[110] W. H. Ramsey Mon. Not. Roy. Astron. Soc. 110 (1950) 325.

[111] M. J. Lighthill Mon. Not. Roy. Astron. Soc. 110 (1950) 339.

[112] B. Kämpfer Phys. Lett. 101B (1981) 366-368.

[113] A. B. Migdal, A. I. Chernoutsan, and I. N. Mishustin Phys. Lett. 83B (1979) 158.

[114] H. Dimmelmeier, M. Bejger, P. Haensel, and J. L. Zdunik Mon. Not. Roy. Astron. Soc. 396 (2009) 2269-2288, arXiv:0901.3819 [astro-ph.SR].

[115] P. Haensel and M. Proszynski Astrophys.J. 258 (1982) 306-320.

[116] I. Mishustin, M. Hanauske, A. Bhattacharyya, L. Satarov, H. Stoecker, et al. Phys.Lett. B552 (2003) 1-8, arXiv:hep-ph/0210422 [hep-ph].

[117] V. Usov Phys.Rev.Lett. 80 (1998) 230-233, arXiv:astro-ph/9712304 [astro-ph].

[118] V. V. Usov Astrophys.J. 559 (2001) L135, arXiv:astro-ph/0107020 [astro-ph].

[119] V. V. Usov Astrophys.J. 550 (2001) L179, arXiv:astro-ph/0103361 [astro-ph]. 
[120] K. Cheng and T. Harko Astrophys.J. 596 (2003) 451-463, arXiv:astro-ph/0306482 [astro-ph].

[121] C. Vogt, R. Rapp, and R. Ouyed Nucl.Phys. A735 (2004) 543-562, arXiv:hep-ph/0311342 [hep-ph]

[122] N. K. Glendenning, C. Kettner, and F. Weber Astrophys. J. 450 (1995) 253-261.

[123] N. K. Glendenning and F. Weber Astrophys. J. 400 (1992) 647-658.

[124] J. Provencal, H. Shipman, E. Hog, and P. Thejll Astrophys.J. 494 (1998) 759-767.

[125] R. P. Negreiros, F. Weber, M. Malheiro, and V. Usov Phys.Rev. D80 (2009) 083006, arXiv:0907.5537 [astro-ph.SR].

[126] A. Brillante and I. N. Mishustin Europhys.Lett. 105 (2014) 39001, arXiv:1401.7915 [astro-ph.SR].

[127] R. Picanco Negreiros, I. N. Mishustin, S. Schramm, and F. Weber Phys.Rev. D82 (2010) 103010, arXiv:1008.0277 [astro-ph.HE].

[128] R. Xu, S. Bastrukov, F. Weber, J. Yu, and I. Molodtsova Phys.Rev. D85 (2012) 023008, arXiv:1110.1226 [astro-ph.HE].

[129] B. Niebergal, R. Ouyed, R. Negreiros, and F. Weber Phys.Rev. D81 (2010) 043005, arXiv:0906.3043 [astro-ph.HE].

[130] F. Weber, R. Negreiros, P. Rosenfield, and M. Stejner Prog.Part.Nucl.Phys. 59 (2007) 94-113, arXiv:astro-ph/0612054 [astro-ph].

[131] B. Golf, J. Hellmers, and F. Weber Phys.Rev. C80 (2009) 015804, arXiv:0906.2427 [astro-ph.SR].

[132] N. Andersson Astrophys. J. 502 (1998) 708-713, arXiv:gr-qc/9706075.

[133] L. Lindblom, B. J. Owen, and S. M. Morsink Phys.Rev.Lett. 80 (1998) 4843-4846, arXiv:gr-qc/9803053 [gr-qc].

[134] J. L. Friedman and S. M. Morsink Astrophys.J. 502 (1998) 714-720, arXiv:gr-qc/9706073 [gr-qc]

[135] P. Jaikumar, G. Rupak, and A. W. Steiner Phys. Rev. D78 (2008) 123007, arXiv:0806.1005 [nucl-th].

[136] G. Rupak and P. Jaikumar Phys.Rev. C82 (2010) 055806, arXiv:1005.4161 [nucl-th].

[137] G. Rupak and P. Jaikumar arXiv:1209.4343 [nucl-th].

[138] B. A. Sa'd arXiv:0806.3359 [astro-ph]. 
[139] M. Mannarelli, C. Manuel, and B. A. Sa'd Phys. Rev. Lett. 101 (2008) 241101, arXiv:0807.3264 [hep-ph].

[140] J. Staff, B. Niebergal, and R. Ouyed Mon. Not. Roy. Astron. Soc. 391 (2008) 178-182, arXiv:0711.3005.

[141] P. Jaikumar, B. S. Meyer, K. Otsuki, and R. Ouyed Astron.Astrophys. 471 (2007) 227-236, arXiv:nucl-th/0610013 [nucl-th].

[142] R. Ouyed and D. Leahy Res.Astron.Astrophys. 13 (2013) 1202-1206, arXiv:1202.2400 [astro-ph.HE].

[143] B.-A. Li Nucl.Phys. A708 (2002) 365-390, arXiv:nucl-th/0206053 [nucl-th].

[144] FOPI Collaboration, Y. Leifels et al. Phys.Rev.Lett. 71 (1993) 963-966.

[145] P. Russotto, P. Wu, M. Zoric, M. Chartier, Y. Leifels, et al. Phys.Lett. B697 (2011) 471-476, arXiv:1101.2361 [nucl-ex],

[146] M. Cozma Phys.Lett. B700 (2011) 139-144, arXiv:1102.2728 [nucl-th].

[147] J. M. Lattimer and Y. Lim Astrophys.J. 771 (2013) 51, arXiv:1203.4286 [nucl-th].

[148] KaoS Collaboration, C. Sturm et al. Phys. Rev. Lett. 86 (2001) 39-42, nucl-ex/0011001.

[149] C. Fuchs, A. Faessler, E. Zabrodin, and Y.-M. Zheng Phys. Rev. Lett. 86 (2001) 1974-1977, nucl-th/0011102.

[150] C. Hartnack, H. Oeschler, and J. Aichelin Phys. Rev. Lett. 96 (2006) 012302, nucl-th/0506087.

[151] I. Sagert, L. Tolos, D. Chatterjee, J. Schaffner-Bielich, and C. Sturm Phys.Rev. C86 (2012) 045802, arXiv:1111.6058 [astro-ph.SR].

[152] P. Braun-Munzinger and J. Stachel J.Phys. G28 (2002) 1971-1976. arXiv:nucl-th/0112051 [nucl-th].

[153] J. Cleymans and K. Redlich Phys.Rev. C60 (1999) 054908, arXiv:nucl-th/9903063 [nucl-th].

[154] O. Buss, T. Gaitanos, K. Gallmeister, H. van Hees, M. Kaskulov, et al. Phys.Rept. 512 (2012) 1-124, arXiv:1106.1344 [hep-ph].

[155] J. M. Lattimer Ann.Rev.Nucl.Part.Sci. 62 (2012) 485-515, arXiv:1305.3510 [nucl-th].

[156] D. B. Blaschke, H. A. Grigorian, D. E. Alvarez-Castillo, and A. S. Ayriyan arXiv:1402.0478 [astro-ph.HE]

[157] E. S. Fraga, A. Kurkela, and A. Vuorinen arXiv:1311.5154 [nucl-th]. 
[158] A. Kurkela, E. S. Fraga, J. Schaffner-Bielich, and A. Vuorinen arXiv:1402.6618 [astro-ph.HE]. 